\title{
On Soft Metric Spaces
}

\author{
O. A. Tantawy , R. M. Hassan \\ Department of Mathematics, Faculty of Science, Zagazig University, Zagazig, Egypt. \\ drosamat@yahoo.com \\ Mathematics Department, Faculty of Science, Suez Canal University, Ismailia, Egypt. \\ nouralsabah333@yahoo.com
}

\begin{abstract}
After the famous article of Moldotsove [10] in 1999 which initiate the theory of soft sets as a mathematical theory to deal with the uncertainty problems, many research works in the soft mathematics and its applications in various fields are appeared. In [17], the authers introduced a new definition of the soft metric function using the soft elements. By this definition each soft metric in view of Das and Samanta [6] is also a soft metric in our concept but the converse is not true. In the present paper, some soft topological properties are given in details, namely (soft compactness, soft sequentially compactness, continuity and uniformly continues of soft functions between soft topological spaces). We hope that the findings in thispaper will help researcher enhance and promote the further study on soft topology to carry out a general framework for their applications in practical life.
\end{abstract}

Indexing terms/Keywords: soft set; soft element; soft topology; soft metric; soft compact; soft sequen-

tially compact; $\epsilon-n e t ;$ totally bounded; soft continuity; soft uniformly continous mapping.

SUBJECT CLASSIFICATION: 03E72, 08A72, 54E50

\section{Introduction}

In 2012. Das and S.K. Samanta [6] have introduced the soft metric. In this paper the soft metric function $d$ on $X$ is defined as a metric function on the set of all soft points on $X$ which is indeed the set $E \times X$, since each ordered pair $(e, x) \in E \times X$ represent a soft point $\mathrm{P}_{\mathrm{e}}{ }^{\mathrm{X}}$. Which implies that the soft metric on $\mathrm{X}$ is not more than the usual metric on the product set $E \times X$, which means that the soft dimension in the problem is totally absence. In the previous work [17], we introduce the soft metric function as a metric function on the set of all soft elements of $X$ which are soft sets $(F, A)$ such that $F(e)$ is a singleton set in $X$ for each $e \in A \subset E$, and the empty set otherwise [16]. By this de?nition each soft metric in view of Das and Samanta [6] is also a soft metric in our concept but the converse is not true. In this work, the soft compactness and the sequentially soft compactness are introduced and the relation between them on the soft metric topological spaces is considered as well. The concept of continuity and uniform continuity of soft functions between soft topological spaces is also presented.

\section{Preliminaries:}

In this section, The basic definitions and results of soft set theory which will be needed in the sequel are presented, $[1,10,11,12,13]$. Throughout this study, $X$ refers to an initial universe $P(X)$ is the power set of $X$, $E$ is a set of parameters and $A \subseteq E$.

Definition 2.1. $([10,11,13])$ A soft set $F_{A}$ on the universe $X$ is defined as a set of ordered

Pair

$$
\mathrm{F}_{A}=\left\{\left(\mathrm{e}, \mathrm{F}_{A}(\mathrm{e})\right): \mathrm{e} \in \mathrm{E}, \mathrm{F}_{A}(\mathrm{e}) \in \mathrm{P}(\mathrm{X})\right\}
$$

where $\mathrm{F}_{\mathrm{A}}: \mathrm{E} \rightarrow \mathrm{P}(\mathrm{x})$, such that $\mathrm{F}_{A}(\mathrm{e})=\phi \forall \mathrm{e} \notin \mathrm{A}$, and $\mathrm{A}$ is called the support of $\mathrm{F}_{\mathrm{A}}$. The collection of all soft sets with support $A$ is denoted by $P(X)^{E}{ }_{A}$.

DeFnition 2.2. ([11]) Let $F_{A}=(F, A)$ and $G_{B}=(G, B)$ be two soft sets over $X$

(1) $(F, A)$ is called a soft subset of $(G, B)$ denoted $(F, A) \subseteq(G, B)$ if $F(e) \subseteq G(e) \forall, e \in$

$\mathrm{A}, \mathrm{A} \subseteq \mathrm{B} \subseteq \mathrm{E}$, and they are equals if $(\mathrm{F}, \mathrm{A}) \subseteq(\mathrm{G}, \mathrm{B})$ and $(\mathrm{G}, \mathrm{B}) \subseteq(\mathrm{F}, \mathrm{A})$.

(2) The union denoted by $\mathrm{F}_{A} \square_{B}$ is the soft set $\mathrm{H}_{\mathrm{C}}=(\mathrm{H}, \mathrm{C})$ where $\mathrm{C}=\mathrm{A} \cup \mathrm{B}$ and $\forall \mathrm{C} \in \mathrm{C}$ 
(3) The intersection denoted by $F_{A} \cap_{G}$ is the soft set $H_{C}=(H, C)$, where $C=A \cap B$ and $H(e)=F(e) \cap G(e) \forall e$ $\in C$.

(4) The difference $(H, A)$ denoted by $(F, A) \tilde{-}(G, B)$ is defined by $H(e)=F(e)-G(e) \forall e \in A$.

(5) The complement is denoted by $\left(\mathrm{F}_{\mathrm{A}}\right)^{c} \tilde{\in} P(\mathrm{X})_{A}^{E}$ where $\left(\mathrm{F}_{\mathrm{A}}\right)^{\mathrm{c}}: \mathrm{E} \rightarrow \mathrm{P}(\mathrm{X})$ is a function given

by $F^{c}(e)=X-F(e) \forall e \in E$, clearly $\left(\left(F_{A}\right)^{c}\right)^{c}=F_{A}$.

Definition 2.3. ([11]) $A$ soft set $F_{A}$ over $X$ is called a null soft set with support $A$, denoted by $\phi_{A}$ if $\mathrm{F}(\mathrm{e})=\phi \forall \mathrm{e} \in \mathrm{A}$ and is called absolute soft set with support $\mathrm{A}$ denoted $\mathrm{By} X_{A}$ if $\mathrm{F}(\mathrm{e})=\mathrm{X} \forall \mathrm{e} \in \mathrm{A}, \mathrm{A} \subseteq \mathrm{E}$ clearly $(\tilde{\phi})^{c}=X$ and $(X)^{c}=\tilde{\phi}$.

Definition 2.4. ([15]) Let $P(\mathrm{X})_{E}^{E}$ and $P(\mathrm{Y})_{K}^{K}$ be the families of all soft sets over $\mathrm{X}$ and $\mathrm{Y}$, respectively. The mapping $(\varphi, \psi)$ is called a soft mapping from $X$ to $Y$, where $\phi: X \rightarrow Y$ and $\psi: E \rightarrow K$ are two mappings.

Let $\mathrm{F}_{\mathrm{A}} \tilde{\in} P(\mathrm{X})_{A}^{E}$, the image of $\mathrm{F}_{\mathrm{A}}$ under the soft mapping $(\varphi, \psi)$ is the soft set over $\mathrm{Y}$ denoted

by $(\varphi, \psi)\left(\mathrm{F}_{\mathrm{A}}\right)$ and denoted by

$$
(\varphi, \psi)\left(F_{A}\right)(k)=\left\{\begin{array}{ll}
\bigsqcup_{e \in \psi^{-1}(k) \cap A} \varphi\left(F_{A}(e)\right) \text { if } \psi^{-1}(k) \cap A \neq \phi \\
\phi & \text { otherwise }
\end{array} .\right.
$$

Definition 2.5. ([5],[6]) A soft element with support $A$ is a soft set such that $F(e)=\left\{x_{e}\right.$

\} is a singleton set $\forall \mathrm{e} \in \mathrm{A}$. The collection of all soft elements is denoted by $[\mathrm{x}]_{E}^{A}$ where

$[X]=\{\{X\}: X \in X\} \cup\{\varphi\}$. The collection of all soft elements with support $A$ is sometimes denoted by $S_{e l}(X, A)$.

Definition 2.6. ([17]) $A$ soft singleton with support $A$ is a soft set with support $A$ such that

$\exists x \in X, \mathrm{~F}(\mathrm{e})=\{\mathrm{x}\} \forall \mathrm{e} \in \mathrm{A}, \mathrm{E}(\mathrm{e})=\phi$ for $e \notin A$. The collection of all soft singletons on $\mathrm{X}$ with support $\mathrm{A}$ is denoted by $\{\mathrm{X}\}_{E}^{A}$ and sometimes denoted by $\mathrm{S}_{\mathrm{s}}(\mathrm{X}, \mathrm{A})$.

Definition 2.7. ([6]) A soft point is a soft set with a singleton support and a singleton

image such that $\mathrm{F}(\mathrm{e})=\{\mathrm{x}\}$, for some $\mathrm{e} \in \mathrm{E}$ and is denoted by $P_{e}^{x}$. The collection of all soft points denoted by $E \times X$ or $\mathrm{S}_{\mathrm{p}}(\mathrm{X}, \mathrm{E})$.

Definition 2.8. ([17]) A soft member is a soft set with singleton support and is denoted by $(e, F(e))$. The collection of all soft member is denoted by $\mathrm{P}(\mathrm{X})_{e}^{E}$ or $\mathrm{S}_{\mathrm{m}}(\mathrm{X}, \mathrm{E})_{\mathrm{e}}$.

Remark 2.1. It is clear that: (i) Any soft element i.e any member of $[\mathrm{X}]_{A}^{E}$ is a union of soft points, i.e, members of $A \times X$.

(ii) Any members of $\mathrm{P}(\mathrm{X})_{A}^{E}$ i.e any soft set is a union of soft elements i.e. member of $[\mathrm{x}]_{E}^{A}$ and also of $A \times X$.

For simplicity, we will use the notations $\alpha, \beta, \tilde{\gamma}, \boldsymbol{\lambda}$ to denote the soft elements, for example

$$
\alpha=\left\{\left(\mathrm{e},\left\{\mathrm{x}_{\mathrm{e}}\right\}\right), \mathrm{e} \in \mathrm{A}\right\} \tilde{\in}[\mathrm{X}]_{A}^{E} .
$$


Definition 2.9. ([17]) A soft element $\alpha=\left\{\left(e,\left\{x_{e}\right\}\right), e \in A\right\}$, is said to belongs to a soft set $F_{A}$, denoted by $\alpha \tilde{\in} F_{A}$, if $\left\{x_{e}\right\} \subset F(e) \forall e \in A \subseteq E$.

Definition 2.10. ([17]) Two soft elements $\alpha=\left\{\left(\mathrm{e},\left\{\mathrm{x}_{\mathrm{e}}\right\}\right), \mathrm{e} \in \mathrm{A}\right\}, \beta=\left\{\left(\mathrm{e},\left\{\mathrm{y}_{\mathrm{e}}\right\}\right), \mathrm{e} \in \mathrm{A}\right\}$ are said to be equals if $(\mathrm{e},\{\mathrm{x}$ e $\})=\left(\mathrm{e},\left\{\mathrm{y}\right.\right.$ e $\forall \mathrm{e} \in \mathrm{A}$ i.e. $\mathrm{x}_{\mathrm{e}}=\mathrm{y}_{\mathrm{e}}$. Thus, $\alpha \neq \beta$ iff $\mathrm{xe} \neq \mathrm{y}$ e, for some e $\in A$.

Proposition 2.1. ([17]) (i) Every soft subset $(F, A), A \in E$ can be considered as a union of its soft elements. Specially it can be considered as a union of soft elements all with the same support $A$.

(ii) Every soft element is a union of a collection of one point support soft elements.

Definition 2.11. A soft subset is called uncountable if it is not a union of countable set of soft elements, and it is countable if it is not uncountable .

Definition 2.12. A soft subset is called infinite if it is not a union of a finite collection of soft sets, and it is finite if it is not infinite.

We are going to define and give some properties of soft real numbers

Definition 2.13. ([5]) A soft real set is a parameterized collection of bounded subsets of real numbers, $P_{b}$ (R).The collection of all soft real sets is denoted by $\mathrm{P}_{b}(\mathrm{R})_{A}^{E}$.

Definition 2.14. ([17]) By a soft real number we mean a soft element of real numbers with support $A$ which is a parametrized collection of real numbers denoted by $\tilde{r}$ i.e. $\tilde{r} \tilde{r}[\mathrm{R}]_{A}^{E}$ and is called a soft +ve real number if $\tilde{r} \tilde{\in}\left[\mathrm{R}^{+}\right]_{A}^{E}$ where $\left[\mathrm{R}^{+}\right]=\left\{\{r\}: r \in \mathrm{R}^{+}\right\} \cup\{\varphi\}$.

In the case of the real numbers as a universal set we have the following concepts:

Definition 2.15. ([17]) (i) A soft singleton +ve real number $\tilde{r}$ is a non-negative soft real number with support A such that

$$
\exists r \in R^{+}, \tilde{r}(e)= \begin{cases}\{r\} & \forall e \in A, \\ \{0\} & \text { for } e \notin A\end{cases}
$$

and is denoted by $\{r\}_{A}^{E}$ for some $r \in \mathrm{R}^{+}$.

(ii) A soft point +ve real no. is a non-negative soft real number with a singleton support and singleton image such that $\tilde{r}(\mathrm{e})=\{\mathrm{r}\}, \mathrm{e} \in \mathrm{A}, \mathrm{r} \in \mathrm{R}^{+}$and the collection of all soft points $+\mathrm{ve}$ real numbers is denoted by $\mathrm{A} \times \mathrm{R}$ + .

(iii) A soft element of real number is a soft set $\tilde{r}=\left\{\left(e,\left\{r_{e}\right\}\right): e \in A, r_{e} \in R\right\}$ with support $A \in E$

(iv) A soft member +ve real no is a non-negative soft real number subset with a singleton support and is denoted by $\mathrm{P}\left(\mathrm{R}^{+}\right)_{e}^{E}$.

An ordered relation on the set of all soft real number elements is given in the following definition:

Definition 2.16. ([17]) (i) For two soft real number elements with support $\mathrm{A} \subset \mathrm{E} \tilde{r}, \tilde{l} \tilde{\in}[\mathrm{R}]_{A}^{E} \tilde{r}, \tilde{r}=\left\{\left\{\mathrm{r}_{\mathrm{e}}\right\}, \mathrm{e} \in\right.$ A $\}, \tilde{l}=\left\{\left\{\mathrm{I}_{\mathrm{e}}\right\}, \mathrm{e} \in \mathrm{A}\right\}$,

$$
\tilde{r} \tilde{\leq} \tilde{l} \text { and } \tilde{l} \tilde{\geq} \tilde{r} \text { if } \mathrm{r}_{\mathrm{e}} \leq \mathrm{I}_{\mathrm{e}} \forall \mathrm{e} \in \mathrm{A} .
$$

(ii) For $\left.\left.\tilde{r}=\left\{\left\{\mathrm{r}_{\mathrm{e}}\right\}: \mathrm{e} \in \mathrm{A}\right\}\right\}, \tilde{l}=\left\{\left\{\mathrm{l}_{\mathrm{e}}\right\}: \mathrm{e} \in \mathrm{A}\right\}\right\} \tilde{\in}[\mathrm{R}]_{A}^{E}$ define the operation $\oplus$ on $[\mathrm{R}]_{A}^{E}$ by

$$
\tilde{r} \oplus \tilde{l}=\left\{\left\{\mathrm{r}_{\mathrm{e}}+\mathrm{I}_{\mathrm{e}}\right\}: \mathrm{e} \in \mathrm{A}\right\} \tilde{\in}[\mathrm{R}]_{A}^{E} \text {, }
$$


and the operation $\ominus$ on $[\mathrm{R}]_{A}^{E}$ by

$$
\tilde{r} \ominus \tilde{l}=\left\{\left\{\mathrm{r}_{\mathrm{e}}-\mathrm{I}_{\mathrm{e}}\right\}: \mathrm{e} \in \mathrm{A}\right\} \tilde{\in}[\mathrm{R}]_{A}^{E},
$$

Definition 2.17. ([15]) A soft topology $T$ is a family of soft sets over $X$ satisfying the following properties.

(1) $\widetilde{\phi}, \tilde{\mathrm{X}} \tilde{\in} \tau$,

(2) If $\mathrm{F}_{\mathrm{A}}, \mathrm{G}_{\mathrm{B}} \tilde{\in} \mathcal{\tau}$, then $\mathrm{F}_{\mathrm{A}} \cap_{\mathrm{G}_{\mathrm{B}}} \tilde{\in} \mathcal{\tau}$,

(3) If $\left(\mathrm{F}_{\mathrm{A}}\right)_{\lambda} \tilde{\in} \mathcal{\tau}, \forall \lambda \in \Lambda$, then $\sqcup_{\lambda \in \Lambda}\left(\mathrm{F}_{\mathrm{A}}\right)_{\lambda} \tilde{\in} \mathcal{\tau}$.

The triple $(X, \tau, E)$ is called a soft topological space over $X$. Every member of $\tau$ is called soft open. A soft set $G_{B}$ is called soft closed in $(X, \tau, E)$ if $\left(G_{B}\right)^{c} \tilde{\in} \tau$. The family of all soft closed sets in $\mathrm{X}$ is denoted by $\tau^{\prime}$.

Definition 2.18. ([3]) Let (X, $\tau$,E) be a soft topological space. A subcollection $B$ of $\tau$ is called a base for $\tau$ if every member of $\tau$ can be expressed as a union of members of $B$.

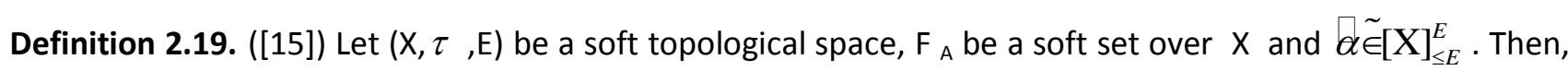
$\mathrm{F}_{\mathrm{A}}$ is called a soft neighborhood of $\alpha$ if there exists a soft open set $\mathrm{G}_{\mathrm{B}}$ such that $\alpha \tilde{e} G_{B} \beth_{F_{A}}$. The neighborhood system of a soft element $\alpha$, denoted by $\mathrm{N}_{\tau}(\alpha)$, is the family of all its neighborhoods.

Definition 2.20. ([19]) Let $\left(\mathrm{X}, \tau\right.$,E) be a soft topological space, $\mathrm{F}_{\mathrm{A}} F_{A} \tilde{\in} P(\mathrm{X})_{A}^{E}$ then $\forall \mathrm{A} \in \mathrm{E}$, we have the following definitions :

(1)The soft closure of $\left(F_{A}\right)$ is the soft set $\overline{(F, A)}=\bigcap_{\left\{G_{A}: G_{A}\right.} \tilde{\in} \tau^{\prime}$ and $\left.F_{A} \tilde{\in} G_{A}\right\}$.

(2) The soft interior of $\left(F_{A}\right)$ is the soft set $(F, A)^{\circ}=\sqsupseteq\left\{G_{A}: G_{A} \in \mathcal{E}\right.$ and $\left.G_{A} \tilde{\in} F_{A}\right\}$.

(3) The soft boundary of $\left(F_{A}\right)$ is the soft set $B(F, A)=\overline{(F, A)} \AA \overline{(F, A)^{c}}$.

Definition 2.21. ([17]) Consider the collection of all soft elements of $X$ with support equal to or contained in E. A mapping $\mathrm{d}_{\mathrm{s}}$ : $[\mathrm{X}]_{\leq E}^{E} \times[\mathrm{X}]_{\leq E}^{E} \rightarrow\left[\mathrm{R}^{+}\right]_{\leq E}^{E}$ is said to be soft metric or distance on the soft set $(\mathrm{X}, \mathrm{E})$ if $\mathrm{d}_{\mathrm{s}}$ satisfies the following conditions

$\left(\mathrm{d}_{1}\right) d_{s}(\alpha, \beta) \tilde{\beta} \tilde{0} \forall \alpha, \tilde{\beta} \tilde{\in}[\mathrm{X}]_{\leq E}^{E}$

$\left(\mathrm{d}_{2}\right) d_{s}(\alpha, \beta)=\tilde{0}$ iff $\varnothing=\beta$

$\left(\mathrm{d}_{3}\right) d_{s}(\alpha, \beta)=d_{s}(\beta, \alpha) \forall \alpha, \tilde{\beta} \tilde{\in}[\mathrm{X}]_{\leq E}^{E}$

$\left(\mathrm{d}_{4}\right) d_{s}(\alpha, \tilde{\gamma}) \tilde{\leq} d_{s}(\alpha, \beta) \oplus d_{s}(\beta, \tilde{\gamma}) \forall \alpha, \beta, \tilde{\gamma} \tilde{\in}[\mathrm{X}]_{\leq E}^{E}$

To have a natural relation between the soft metric structures and the metric structures we reconsider a special case of soft metrics in which the soft distance is defined between soft elements with the same support only.

Definition 2.22. ([17]) A soft metric $d_{s}$ on $[X]_{\leq E}^{E}$ is called equal support soft metric or (E-soft metric) denoted by $\mathrm{d}_{\mathrm{ES}}$ if the conditions $\left(\mathrm{d}_{1}\right),\left(\mathrm{d}_{2}\right),\left(\mathrm{d}_{3}\right),\left(\mathrm{d}_{4}\right)$ in definition are holds for soft elements $\alpha, \beta, \tilde{\gamma}$ with equal supports and $\left(\mathrm{d}_{\mathrm{ES}}(\alpha, \beta)\right)(\mathrm{e})=0 \forall \mathrm{e} \notin$ support $\alpha$. 
In the following proposition we show that a given soft metric on $(X, E)$ can be generate a collection of usual pseudo-metrics or metrics on $\mathrm{X}$.

Proposition 2.2. ([17]) Given a soft metric $d_{S}$ on $(X, E), d_{s}:[X]_{\leq E}^{E} \times[X]_{\leq E}^{E} \rightarrow\left[R^{+}\right]_{\leq E}^{E}$. For every pair of points $(\mathrm{x}, \mathrm{y})$ of $\mathrm{X}$, consider the soft singletons $x, y$ and consider the soft distance between them $\mathrm{d}_{\mathrm{s}}(x, y)=\{(\mathrm{e}$,

$\left.\left.\left\{r_{\mathrm{e}}\right\}\right): \mathrm{e} \in \mathrm{E}\right\}=\tilde{r}$. For every parameter $\mathrm{e} \in \mathrm{E}$ consider the +ve real valued function, $\mathrm{d}_{\mathrm{e}}(\mathrm{x}, \mathrm{y})$ given by $\mathrm{d}_{\mathrm{e}}$ $(x, y)=r_{e}$. Then, $d_{e}$ is a usual pseudo metric on $X$ i.e. $d_{s}$ generate a collection of usual pseudo metrics $\left\{d_{e}\right.$ $: e \in E\}$ on $X$.

Definition 2.23. ([17]) Consider a soft metric space $\left(X, E, d_{S}\right)$ and let $Y_{A}$ be any soft subset of $(X, E)$. The soft diameter of $\mathrm{Y}_{\mathrm{A}}$, denoted by $\mathrm{Sd}\left(\mathrm{Y}_{\mathrm{A}}\right)=\left\{\left(\mathrm{e},\left\{\mathrm{d}_{\mathrm{e}}\right\}\right): \mathrm{e} \in \mathrm{A}\right\} \tilde{\in}\left[\mathrm{R}^{+}\right]_{\leq E}^{E}$ given by

$$
\mathrm{Sd}\left(\mathrm{Y}_{\mathrm{A}}\right)=\sup \left\{\mathrm{d}_{\mathrm{s}}(\alpha, \beta): \not \alpha, \beta \tilde{\beta} \tilde{\in} \mathrm{Y}_{A}^{E}\right\} .
$$

In other word, if $\mathrm{d}_{\mathrm{s}}(\alpha, \beta)=\left\{\left(\mathrm{e},\left\{\mathrm{r}_{\mathrm{e}}(\alpha, \beta)\right\}\right): \mathrm{e} \in \mathrm{E}\right\}$ then,

$$
\begin{gathered}
\operatorname{sd}\left(\mathrm{Y}_{\mathrm{A}}\right)=\left\{\sup \left\{\left(\mathrm{e},\left\{\mathrm{r}_{\mathrm{e}}(\alpha, \beta)\right\}\right): \mathrm{e} \in \mathrm{A}\right\} ; \not \alpha, \beta \tilde{\in}[\mathrm{Y}]_{A}^{E}\right\} \\
=\left\{\left(\mathrm{e},\left\{\sup \left\{\mathrm{r}_{\mathrm{e}}(\alpha, \beta)\right\}: \mathrm{e} \in \mathrm{A}\right\} ; \alpha, \beta \tilde{\tilde{E}}[\mathrm{Y}]_{A}^{E}\right\} .\right.
\end{gathered}
$$

Also, a soft subset $\mathrm{Y}_{A}$ is called bounded if $\exists$ a soft real number $d$ for which $S_{d}\left(Y_{A}\right) \simeq \mathbb{d}$.

Definition 2.24. ([17]) Let $d_{S}$ be a soft metric on $(X, E)$. For any soft element $Q \alpha=\left\{\left(e,\left\{x_{\mathrm{e}}\right\}\right):\right.$ e $\left.\in A\right\} \tilde{\in}[X]_{\leq E}^{E}$ and any +ve soft real number $\tilde{r}=\left\{\left(\mathrm{e},\left\{\mathrm{r}_{\mathrm{e}}\right\}\right): \mathrm{e} \in \mathrm{E}\right\} \tilde{\in}\left[\mathrm{R}^{+}\right]_{E}^{E}$. The soft open ball with center $\alpha$ and radius $\tilde{r}$, denoted by $\operatorname{SB}(\alpha, \tilde{r})$ is defined by

$$
\mathrm{SB}(\alpha, \tilde{r})=\sqcup\left\{\beta: \mathrm{d}_{\mathrm{s}}(\alpha, \beta) \tilde{<} \tilde{r}\right\},
$$

where $\beta=\left\{\left(\mathrm{e},\left\{\mathrm{y}_{\mathrm{e}}\right\}\right): \mathrm{e} \in \mathrm{B}\right\} \tilde{\in}[\mathrm{X}]_{\leq E}^{E}$, where $\mathrm{A}, \mathrm{B}$ are arbitrary subsets of $\mathrm{E}$.

Also the soft closed ball with center $\sim \alpha$ and radius $~ r$ denoted by

$$
\mathrm{SB}_{[}\left[\alpha, \tilde{r}_{]}=\sqcup\left\{\beta: \mathrm{d}_{\mathrm{s}}(\alpha, \beta) \widetilde{z} \tilde{r}\right\} .\right.
$$

Corollary 2.1. ([17]) The collection of all soft open balles in any soft metric space $\left(X, E, d_{s}\right)$ is a base for a soft topology denoted by $\tau_{d_{s}}$ on $(\mathrm{X}, \mathrm{E})$.

De?nition 2.25. ([17]) Let $\left(X, E, d_{s}\right)$ be any soft metric space. A sequence $\left\{\alpha_{n}: n \in N\right\}$ of soft elements is said to be convergent in $\left(\mathrm{X}, \mathrm{E}, \mathrm{d}_{\mathrm{S}}\right)$ if there exists a soft element $\beta$ such that $\lim _{n \rightarrow \infty} d_{S}\left(\alpha_{n}, \beta\right)=\tilde{0}$, i.e. for every soft real number $\tilde{\varepsilon}>\tilde{0}$, there exists a natural number $n_{\varepsilon}$ such that $d_{S}\left(\alpha_{n}, \beta\right) \tilde{\beta} \tilde{\varepsilon} \forall \mathrm{n}>n_{\varepsilon} . \beta$ is called a limit soft element of $\left\{\alpha_{n}\right\}$.

Theorem 2.1. ([17]) Limit of sequence in a soft metric space if exists is unique.

Definition 2.26. ([17]) Let $\left(X, E, d_{S}\right)$ be any soft metric space, $F_{A}$ be any soft subset and $\alpha$ be a soft element of $(\mathrm{X}, \mathrm{E}) . \alpha$ is called a soft limit element of $\mathrm{F}_{\mathrm{A}}$ if $(\mathrm{SB}(\alpha, \tilde{\varepsilon}) \tilde{-}\{\alpha\}) \cap_{\mathrm{F}_{\mathrm{A}}} \neq \tilde{\phi}$ for every soft real number $\tilde{\varepsilon}>\tilde{\varepsilon}$. The union of all the soft limit elements of $\mathrm{F}_{\mathrm{A}}$ is called the derived set of $\mathrm{F}_{\mathrm{A}}$ denoted by $F_{A}^{d}$ 
Definition 2.27. ([17]) A sequence $\left\{\alpha_{n}: n \in N\right\}$ of soft elements in a soft metric space $\left(X, E, d_{s}\right)$ is called a cauchy sequence if for every soft real number $\tilde{\varepsilon} \tilde{\varepsilon}\left[\mathrm{R}^{+}\right]_{\leq E}^{E}$, there exists a natural number $n_{\varepsilon}$ such that $\mathrm{d}_{\mathrm{s}}$ $\left(\alpha_{\mathrm{n}}, \alpha_{\mathrm{m}}\right) \tilde{\leq} \tilde{\varepsilon} \forall \mathrm{n}, \mathrm{m} \geq n_{\varepsilon}$.

Definition 2.28. ([17]) A soft metric space $\left(X, E, d_{S}\right)$ (respectively a soft subset $Y_{A}$ of $(X, E)$ ) is called complete metric space if every cauchy sequence in $(X, E)$ (respectively in $Y_{A}$ ), converges to a soft element in $(X, E)$ (respectively in $\mathrm{Y}_{\mathrm{A}}$ ).

\section{Soft Compactness in Soft Metric Spaces}

In this section, we introduce the concept of soft compactness and sequentially soft compactness in the soft metric spaces.

De?nition 3.1. Let $\left(\mathrm{X}, \mathrm{E}, \tau_{d_{S}}\right)$ be a soft topological space and $\mathrm{W}_{\mathrm{B}} \subseteq \mathrm{X}$

1. A collection $\left\{\left(F_{A}\right) \alpha: \alpha \in \Gamma\right\}$ of soft open sets is called a soft open cover of $X$, if it is

satisfies $\mathrm{W}_{\mathrm{B}} \subset \cup_{\alpha} F_{A_{\alpha}}$. A finite subcollection of a soft open $\operatorname{cover}\left\{\left(\mathrm{F}_{\mathrm{A}}\right) \alpha: \alpha \in \Gamma\right\}$ of $\mathrm{W}_{\mathrm{B}}$ is called a finite subcover of $\left\{\left(\mathrm{F}_{\mathrm{A}}\right) \alpha: \alpha \in \Gamma\right\}$.

2. $W_{B}$ is called soft compact if every soft open cover of $W_{B}$ has a finite subcover.

Definition 3.2. Let $\left(X, E, d_{S}\right)$ be a soft metric space, $\left(X, E, d_{S}\right)$ is called a soft sequential compact metric space if every soft sequence has a soft subsequence that converges in $X$.

In the following, we give the relation between an equal support soft compact space (soft sequentially compact soft metric space) and the parametrized collection of compact spaces (sequentially compact metric spaces) generated by it.

Proposition 3.1. If $\left(X, E, d_{E S}\right)$ is a soft sequentially compact soft metric space, then $\left(X, d_{e}\right)$ is sequentially compact metric space for each e $\in E$, where $\left\{\left(X, d_{e}\right): e \in E\right\}$ is a parametrized collection of metric spaces generated by $\left(X, E, d_{E S}\right)$.

Proof. Let $\left(X, E, d_{E S}\right)$ be an equal support soft sequentially compact soft metric space and let $\left\{\left(X, d_{e}\right): e \in E\right\}$ be the parametrized collection of metric spaces generated by $\left(X, E, d_{E S}\right)$ (proposition (2.2)).

If $\left\{\alpha_{n}^{e}: \mathrm{n} \in \mathrm{N}\right\}$ be a convergent sequence in $\left(\mathrm{X}, \mathrm{d}_{\mathrm{e}}\right)$ for each $\mathrm{e} \in \mathrm{E}$, then for each $\mathrm{n} \in \mathrm{N}, \alpha_{n}=\left\{\left(\mathrm{e}, \alpha_{n}^{e}\right):\right.$ e $\in$ E\} is a soft element in $\left(\mathrm{X}, \mathrm{E}, \mathrm{d}_{\mathrm{ES}}\right)$, where $\alpha_{n}(\mathrm{e})=\alpha_{n}^{e}$ and then $S=\left\{\alpha_{n}: \mathrm{n} \in \mathrm{N}\right\}$ is a convergent sequence of equal support soft elements in $\left(\mathrm{X}, \mathrm{E}, \mathrm{d}_{\mathrm{ES}}\right)$. Consequently, $S=\left\{\alpha_{n}:\right.$ e $\left.\in \mathrm{N}\right\}$ has a subsequence of equal support soft elements $S_{S}=\left\{\alpha_{n_{k}}: \mathrm{n}_{\mathrm{k}} \in \mathrm{N}\right\}$ which converges in $\left(\mathrm{X}, \mathrm{E}, \mathrm{d}_{\mathrm{ES}}\right)$. This implies that for each e $\in \mathrm{E}$, the sequence $\left\{\alpha_{n}^{e}: \mathrm{n} \in \mathrm{N}\right\}$ has a subsequence $\left\{\alpha_{n_{k}}^{e}: \mathrm{n} \in \mathrm{N}\right\}$ that converges in $\left(\mathrm{X}, \mathrm{d} \mathrm{e}_{\mathrm{e}}\right)$.

Proposition 3.2. If $\left(X, E, \tau_{d_{S}}\right)$ is a soft compact, then $\left(X, d_{e}\right)$ is compact for each e $\in E$, where $\left\{\left(X, d_{e}\right): e \in E\right\}$ is a parametrized collection of metric spaces generated by $\left(X, E, d_{E S}\right)$.

Proof. Straightforwards

Proposition 3.3. Let $\left(X, E, d_{S}\right)$ be any soft metric space. (X,E, $\left.d_{E S}\right)$ is soft sequentially compact soft metric space then every infinite soft subset $F_{A}$ has a soft limit element.

Proof. $=\Rightarrow$ Let $F_{A}$ be an infinite soft subset and $\left\{\alpha_{n}: n \in N\right\}$ be a sequence of soft elements in $F_{A}$. Then $\left\{\alpha_{n}: \mathrm{n} \in \mathrm{N}\right\}$ has a convergent subsequence of soft element $\left\{\alpha_{n_{k}}: \mathrm{n}_{\mathrm{k}} \in \mathrm{N}\right\}$. Assume that $\left\{\alpha_{n_{k}}: \mathrm{n}_{\mathrm{k}} \in \mathrm{N}\right\}$ converges to a soft element $\alpha$. Since for every soft real number $\tilde{\varepsilon} \sim \tilde{0}$ 


$$
\tilde{\phi} \neq \mathrm{SB}(\alpha, \tilde{\varepsilon}) \varnothing\left\{\alpha_{n_{k}}: \mathrm{n}_{\mathrm{k}} \in \mathrm{N}\right\} \subset \mathrm{SB}(\alpha, \tilde{\varepsilon}) \varnothing \mathrm{F}_{\mathrm{A}},
$$

$\alpha$ is a soft limit element in $F_{A}$.

Definition 3.3. Let $\left(X, E, d_{S}\right)$ be a soft metric space and let $\tilde{\varepsilon}$ be a soft real number. A collection of soft elements $\psi=\left\{\alpha_{\lambda}: \lambda \in \Lambda\right\}$ is called a soft $\tilde{\varepsilon}$-net in $\left(\mathrm{X}, \mathrm{E}, \mathrm{d}_{\mathrm{S}}\right)$ if $X=\square_{\lambda \in \Lambda} \mathrm{SB}\left(\alpha_{\lambda}, \tilde{\varepsilon}_{)}=\square_{\alpha \tilde{\alpha} F_{A}} \mathrm{SB}(\alpha, \tilde{\varepsilon})\right.$, where $\mathrm{F}_{\mathrm{A}}=\sqcup \psi=\square\left\{\alpha_{\lambda}: \lambda \in \Lambda\right\}$.

Definition 3.4. A soft metric space $\left(X, E, d_{S}\right)$ is called totally bounded if for each soft real number $\tilde{\varepsilon} \sim \tilde{\varepsilon} \tilde{0}$, there exists a finite soft $\tilde{\varepsilon}$-net in $\left(\mathrm{X}, \mathrm{E}, \mathrm{d}_{\mathrm{s}}\right)$.

Lemma 3.1. Let $(X, E, d S)$ be a totally bounded soft metric space and $\tilde{\varepsilon}$ be any fixed soft real number, $F_{A}$ be an infinite soft subset. Then there is an infinite soft subset $G_{B}$ of $F_{A}$ such that $\operatorname{Sd}\left(G_{B}\right) \widetilde{<} \tilde{\varepsilon}$.

Proof. Let $\left(X, E, d_{S}\right)$ be a totally bounded soft metric space and let $\tilde{\varepsilon}$ be a soft real number, then there exists a finite soft $\frac{\varepsilon}{2}$-net $\psi=\left\{\alpha_{i}: \mathrm{i}=1,2, \ldots, \mathrm{n}\right\}$ in $\left(\mathrm{X}, \mathrm{E}, \mathrm{d}_{\mathrm{s}}\right)$, where $X=\square_{\mathrm{i}} \mathrm{SB}\left(\alpha_{i}, \frac{\varepsilon}{2}\right)$. Consequently, for any infinite soft subset $\mathrm{F}_{\mathrm{A}}$ of $X, \mathrm{~F}_{\mathrm{A}}=\mathrm{F}_{\mathrm{A}} \cap X=\square_{i=1}^{n} \mathrm{FA} \cap \mathrm{SB}\left(\alpha_{i}, \frac{\varepsilon}{2}\right)$.

Since $\mathrm{F}_{\mathrm{A}}$ is infinite soft subset then, $\exists \mathrm{j} \in\{1,2, \ldots, \mathrm{n}\}$ for which $\mathrm{G}_{\mathrm{B}}=\mathrm{F}_{\mathrm{A}} \cap_{\mathrm{SB}}\left(\alpha_{j}, \frac{\varepsilon}{2}\right)$ is an infinite soft subset. So, $\operatorname{Sd}\left(\mathrm{G}_{\mathrm{B}}\right) \tilde{<\tilde{\varepsilon}}$.

Theorem 3.1. Let $(X, E, d S)$ be a soft metric space. $(X, E, d S)$ is totally bounded if and only if every soft sequence has a Cauchy soft subsequence in $X$.

Proposition 4.2. Every soft uniformly continues mapping is soft continues.

Proof. Straightforwards

\section{Conclusion}

In the previous paper, soft metric function is introduced using the soft elements of the space, examples and many properties are studied. Also, some soft topological properties are given. In this paper, a sequel of the previous work is introduced such as soft compactness and soft sequentially compact soft metric space. Later we give the concepts of $\sim \epsilon-$ net and totally bounded soft metric spaces. Finally, we present the concept of soft continuity and soft uniformly continues of soft mappings and we investigate some important theorems in details.

Acknowledgement: Our thanks to the experts who have contributed towards development of the template.

\section{References}

[1] M. I. Ali, M. Shabir, M. Naz, Algebraic structures of soft sets associated with new operations, Computer and Mathematics with Applications, vol. 61, 2647 - 2654, (2011).

[2] B. Ahmed and S. Hussain, On Some Structures of Soft Topology, Mathematical Sciences, 6 - 64, (2012).

[3] A. Ayg?no?lu and H. Ayg?n, Some notes on soft topological spaces, Neural Comput Applic, vol. 21, s113s119, (2012). 
[4] N. Cagman, S. Karatas, S. enginoglu, Soft Topology, Computer and Mathematics with Applications, vol. 62, 351 - 358, (2011).

[5] S. Das, S. K. Samanta, Soft Real Sets, Soft Real Numbers and Their Properties, J. Fuzzy Math., 551-576, (2012).

[6] S. Das, S. K. Samanta, Soft metric, Annals of Fuzzy Mathematics and Information, 1 - 18, (2012).

[7] D. N. Georgiou, A. C. Megaritis and V. I. Petropoulos, On soft topological spaces,

Applied Mathematics and Information Sciences, vol. 7, no. 5, 1889 - 1901, (2013).

[8] H. Hazra, P. Majumdar, S. K. Samanta, Soft Topology, Fuzzy Inf. Eng., 105 - 115, (2012).

[9] S. Hussain, B. Ahmed, Some properties of soft topological spaces, Computer and Math-ematics with Applications, vol. 62, 4058 - 4067, (2011).

[10] D. Molodotsov, Soft Set Theory - First Results, Computer and Mathematics with Applications, vol. 37, 19 - 31, (1999).

[11] P. K. Maji, Rbiswas and A. R. Roy, Soft Set Theory, Computers and Mathematics with Applications, 555 562, (2003).

[12] W. K. Min, A note on soft topological spaces, Computer and Mathematics with Appli-cations, vol. 62, 3524 - 3528, (2011).

[13] D. Pei, D. Miao, From Soft Sets to Information System, IEEE, 617 - 621, (2005).

[14] W. Rong, The countabilities of Soft Topological Spaces, World Academy of Science, Engineering and Technology, vol. 6, (2012).

[15] M. Shabir and M. Naz, On soft topological Spaces, Comput. Math. Appl., vol. 61, 1786-1799, (2011).

[16] O. A. Tantawy, M. Hosny, Ordered soft topological spaces, submmited for publication.

[17] O. A. Tantawy, R. M. Hassan, Soft Metric Spaces, 5th International Conference on Mathematics and Information Sciences, (2016) .

[18] B. P. Varol, H. Aygun, On Soft Hausdor? Spaces, Annals of Fuzzy and Information, vol. 5, no. 1, 15 - 24, (2013).

[19] Zorlutuna, M. Akdag, W. K. Min, S. Atmaca, Remarks on soft topological spaces, Annals of Fuzzy and Information, vol. 3, no. 2, 171 - 185, (2012). 